\title{
Nonlinear Observer Design for One-Sided Lipschitz Generalized System
}

\author{
Yunan Zhao ${ }^{1, a}$ and JunguoLu ${ }^{1, b}$ \\ ${ }^{1}$ School of Electronics, Information and Electrical Engineering, Shanghai Jiao Tong University, \\ Shanghai200240, China;
}

a284521832@sjtu.edu.cn, bjglu@sjtu.edu.cn

Keywords:Generalized system,one-sided Lipschitz condition,observer design, LMI, LME.

\begin{abstract}
State estimation of systems satisfying some special nonlinearities have been important topics in nonlinear system theory. In this paper, we discuss the problem ofobserver design for one-sided Lipschitz nonlinear generalized systems basedon generalized quadratic stability and Lyapunov function, by using the linear matrix inequality (LMI) andlinear matrix equality (LME) approach, we propose some sufficient conditions for thegeneralized quadratic stability of one-sided Lipschitz generalized systems, which ensure that the observererror dynamics is generalized quadratically stable. Simulation results on one example are given to illustratethe effectiveness and advantages of the proposed design.
\end{abstract}

\section{Introduction}

During the last decades, enormous efforts are put into the study of nonlinear system[1-2], and the observer design problem for nonlinear systems satisfying a Lipschitz conditionhas motivated plenty of interests in nonlinear system theory. In [3], Thau presented asufficient condition which ensures the asymptotical stability of observer error dynamics.Raghavan and Hedrick in [4] derived a constructive design by iteratively solving a series of Riccati equations. What's more, a lot of researchers studied observer design forLipschitz systems with different methods.

It is interesting that the Lipschitz constants of such functions are often region based,with the operating region enlarged, the Lipschitz constants usually increase dramatically.However, most of the existing approaches can only deal with the situation when Lipschitzconstants are small, in other words, these techniques can not find solutions with largeLipschitz constants. For the purpose of overcoming this drawback, Lipschitz continuityis generalised to a less restrictive condition known as one-sided Lipschitz continuity,which has been extensively applied to the stability analysis and numerical analysis ofordinary differential equations. Compared with the Lipschitz constant, the one-sidedLipschitz constant is much more suited for estimating the influence of nonlinear partbecause it possesses inherent advantages with respect to conservativeness. Inspired bythe advances of the one-sided Lipschitz constant, various methods are considered andapplied to design observers of nonlinear systems, more results on this problem can befound in [5-6].

In this paper, we extend the nonlinear observer design method on generalized systems. We present some sufficient conditions which ensure thatthe observer error dynamics isgeneralized quadratic stability stable. A simulative example is included to illustrate the effectiveness and advantages of the proposed methods. The remainder of the paper is organized as follows: Section 2 states the problem we deal with and introduce some basicdefinitions. Section 3, whichcontains the main results, presents LMI/LME-based observer design approachesfor one-sided Lipschitz generalized systems. Then section 4 provides an illustrative example. In the end section 5 gives the conclusion of this paper.

\section{Problem statement}

In this section, we consider the following nonlinear generalised system

$$
\left\{\begin{array}{c}
E \dot{x}(t)=A x(t)+\phi(x, u) \\
y(t)=C x(t)
\end{array}\right.
$$


where $E \in \mathbb{R}^{n \times n}$ is a singular matrix known as the state matrix, $x \in \mathbb{R}^{n}$ is the state, $u \in \mathbb{R}^{m}$ is the control input, $y \in \mathbb{R}^{p}$ is the measured output, $A \in \mathbb{R}^{n \times n}, C \in \mathbb{R}^{n \times p}$ are const matrices. The nonlinear function $\phi(x, u)$ is continuous with respect to both $x$ and $u$.

Definition $1^{[7]}$. The nonlinear function $\phi(x, u)$ issaid to be 'one-sided Lipschitz' in a region $D$ if there exists $\rho \in \mathbb{R}$ such that $\forall x_{1}, x_{2} \in D$,

$<\phi\left(x_{1}, u^{*}\right)-\phi\left(x_{2}, u^{*}\right), x_{1}-x_{2}>\leq \rho\left\|x_{1}-x_{2}\right\|^{2}$.

Definition $2^{[7]}$. The nonlinear function $\phi(x, u)$ issaid to be 'quadratically inner-bounded' in a region $\widetilde{D}$ if there exists $\beta, \gamma \in \mathbb{R}$ such that $\forall x_{1}, x_{2} \in \widetilde{D}$,

$\left\|\phi\left(x_{1}, u\right)-\phi\left(x_{2}, u\right)\right\|^{2} \leq \beta\left\|x_{1}-x_{2}\right\|^{2}+\gamma<\phi\left(x_{1}, u\right)-\phi\left(x_{2}, u\right), x_{1}-x_{2}>$.

Definition $3^{[8]}$.System (1) issaid to be ' generalized quadratically stable'if there exists a matrix Psuch that

$$
\begin{gathered}
E^{T} P=P^{T} E \geq 0, \\
{[A x(t)+\phi(x, u)]^{T} P x(t)+x^{T}(t) P^{T}[A x(t)+\phi(x, u)]<0 .}
\end{gathered}
$$

For system (1), we consider a full-order observer of the following form

$$
E \dot{\hat{x}}(t)=A \hat{x}(t)+\phi(\hat{x}, u)+L(y-C \hat{x})
$$

where $L$ is a gain matrix for the observer. Denote $e(t)=x(t)-\hat{x}(t), \phi=\phi(x, u), \hat{\phi}=$ $\phi(\hat{x}, u)$, Thenthe observer error dynamics is given by

$$
E \dot{e}(t)=(A-L C) e(t)+\phi-\hat{\phi}
$$

assuming that $\phi$ is one-sided Lipschitz and quadratically inner-bounded. Let $\tilde{\phi}=\phi-\hat{\phi}$, with definition 2 and definition 3 , we obtain

$$
\begin{gathered}
e^{T}(t) \tilde{\phi} \leq \rho e^{T}(t) e(t) \\
\tilde{\phi}^{T} \tilde{\phi} \leq \beta e^{T}(t) e(t)+\gamma e(t) \widetilde{\phi} .
\end{gathered}
$$

Our design goal is to find an observer gain matrix $L$ for system (1), with $\phi$ satisfying conditon (4) and (5), such that the observer error dynamics (3) is generalized quadraticallystable.

\section{Main Results}

Theorem1. Assume that $\phi$ in system (1) satisfies the conditions (4) and (5), the state observer for system (1) holds the form of (2), then the observer error dynamics (3) is generalized quadraticallystable, if there existsan invertible matrix $P$, a matrix $R$ and 2 scalars $\varepsilon_{1}>0, \varepsilon_{2}>$ 0 ,such that the following LMI and LME are feasible:

$$
\begin{gathered}
E^{T} P=P^{T} E \geq 0, \\
{\left[\begin{array}{cc}
A^{T} P+P^{T} A-C^{T} R-R^{T} C+\varepsilon_{1} \rho I+\varepsilon_{2} \beta I & P^{T}-\frac{\varepsilon_{1} I}{2}+\frac{\varepsilon_{2} \gamma I}{2} \\
P-\frac{\varepsilon_{1} I}{2}+\frac{\varepsilon_{2} \gamma I}{2} & -\varepsilon_{2} I
\end{array}\right]<0 .}
\end{gathered}
$$

If (6) and (7) are feasible, then the gain matrixis given by $L=P^{-T} R^{T}$.

Proof. Suppose there existsan invertible matrix $P$ satisfying $E^{T} P=P^{T} E \geq 0$, select Lyapunov function $V(t)=e^{T}(t) E^{T} P e(t)$, then

$$
\begin{aligned}
\dot{V}(t)=e^{T}(t)(A-L C)^{T} P e(t)+\tilde{\phi}^{T} P e(t) & +e^{T}(t) P^{T}(A-L C) e(t)+e^{T}(t) P^{T} \tilde{\phi} \\
& =\left[\begin{array}{c}
e(t) \\
\tilde{\phi}
\end{array}\right]^{T}\left[\begin{array}{cc}
A^{T} P+P^{T} A-C^{T} L^{T} P-P^{T} L C & P^{T} \\
P & 0
\end{array}\right]\left[\begin{array}{c}
e(t) \\
\tilde{\phi}
\end{array}\right] .
\end{aligned}
$$

For any positive scalars $\varepsilon_{1}, \varepsilon_{2}$, with condition (4) and (5), we obtain

$$
\varepsilon_{1}\left[\begin{array}{c}
e(t) \\
\tilde{\phi}
\end{array}\right]^{T}\left[\begin{array}{cc}
\rho I & -\frac{I}{2} \\
-\frac{I}{2} & 0
\end{array}\right]\left[\begin{array}{c}
e(t) \\
\tilde{\phi}
\end{array}\right]+\varepsilon_{2}\left[\begin{array}{c}
e(t) \\
\tilde{\phi}
\end{array}\right]^{T}\left[\begin{array}{cc}
\beta I & \frac{\gamma I}{2} \\
\frac{\gamma I}{2} & -I
\end{array}\right]\left[\begin{array}{c}
e(t) \\
\tilde{\phi}
\end{array}\right] \geq 0
$$

Thus, adding the left terms of (7) to (6), we obtain

$$
\dot{V}(t) \leq\left[\begin{array}{c}
e(t) \\
\tilde{\phi}
\end{array}\right]^{T}\left[\begin{array}{cc}
A^{T} P+P^{T} A-C^{T} L^{T} P-P^{T} L C+\varepsilon_{1} \rho I+\varepsilon_{2} \beta I & P^{T}-\frac{\varepsilon_{1} I}{2}+\frac{\varepsilon_{2} \gamma I}{2} \\
P-\frac{\varepsilon_{1} I}{2}+\frac{\varepsilon_{2} \gamma I}{2} & -\varepsilon_{2} I
\end{array}\right]\left[\begin{array}{c}
e(t) \\
\tilde{\phi}
\end{array}\right] .
$$


Let $R=L^{T} P$, then it follows from (8) that $\dot{V}(t)<0$ provided thatthe following LMI holds a feasible solution:

This ends the proof.

$$
\left[\begin{array}{cc}
A^{T} P+P^{T} A-C^{T} R-R^{T} C+\varepsilon_{1} \rho I+\varepsilon_{2} \beta I & P^{T}-\frac{\varepsilon_{1} I}{2}+\frac{\varepsilon_{2} \gamma I}{2} \\
P-\frac{\varepsilon_{1} I}{2}+\frac{\varepsilon_{2} \gamma I}{2} & -\varepsilon_{2} I
\end{array}\right]<0 .
$$

\section{Numercial example}

Design a nonlinear observer (2) for the generalizd system (1) with

$$
\begin{gathered}
\mathrm{A}=\left[\begin{array}{rccc}
1.26 & -48.6 & 0 & 0 \\
-10 & 0 & -1 & 1.33 \\
0 & 1.6 & 0 & -21.6 \\
0 & 0 & -1.95 & 1
\end{array}\right], \mathrm{C}=\left[\begin{array}{llll}
1 & 0 & 0 & 0 \\
0 & 1 & 0 & 0
\end{array}\right], \\
\mathrm{E}=\left[\begin{array}{llll}
1 & 0 & 0 & 0 \\
0 & 1 & 0 & 0 \\
0 & 0 & 0 & 0 \\
0 & 0 & 0 & 0
\end{array}\right], \phi(x, u)=\left[\begin{array}{c}
\sin \left(x_{1}\right) \\
0 \\
0 \\
0
\end{array}\right] .
\end{gathered}
$$

It is easy to find that $\rho=1, \beta=1, \gamma=0$.Theorem 1 can be applied to design a full-order observer, which gives $\varepsilon_{1}=11244.1, \varepsilon_{2}=15623.852$,

$$
\begin{aligned}
& \mathrm{P}=\left[\begin{array}{cccc}
9745.9 & -314.9 & 0 & 0 \\
-314.9 & 9732.49 & 0 & 0 \\
-2.3 & 3.8 & 197.9 & 1107.8 \\
-2.5 & 13.4 & 12984.0 & 3355.5
\end{array}\right], \\
& R=\left[\begin{array}{rlll}
36475.1 & 0 & 318.9 & -371.7 \\
-571579.3 & 36352.4 & -9436.2 & 14646.9
\end{array}\right] \text {. }
\end{aligned}
$$

Then we get the observer gain matrix

$$
L=P^{-T} R^{T}=\left[\begin{array}{cc}
3.7464 & -58.5853 \\
0.1213 & 1.8345 \\
-0.4299 & 16.1692 \\
0.0311 & -0.9733
\end{array}\right]
$$

We use Matlab/simulink tool for simulation, figure 1 displays the state of the observer error dynamicsfor system (1), which shows all the state $e_{1}, e_{2}, e_{3}, e_{4}$ are generalized quadratically stable. The simulation results verifythe effectiveness of the proposed design.
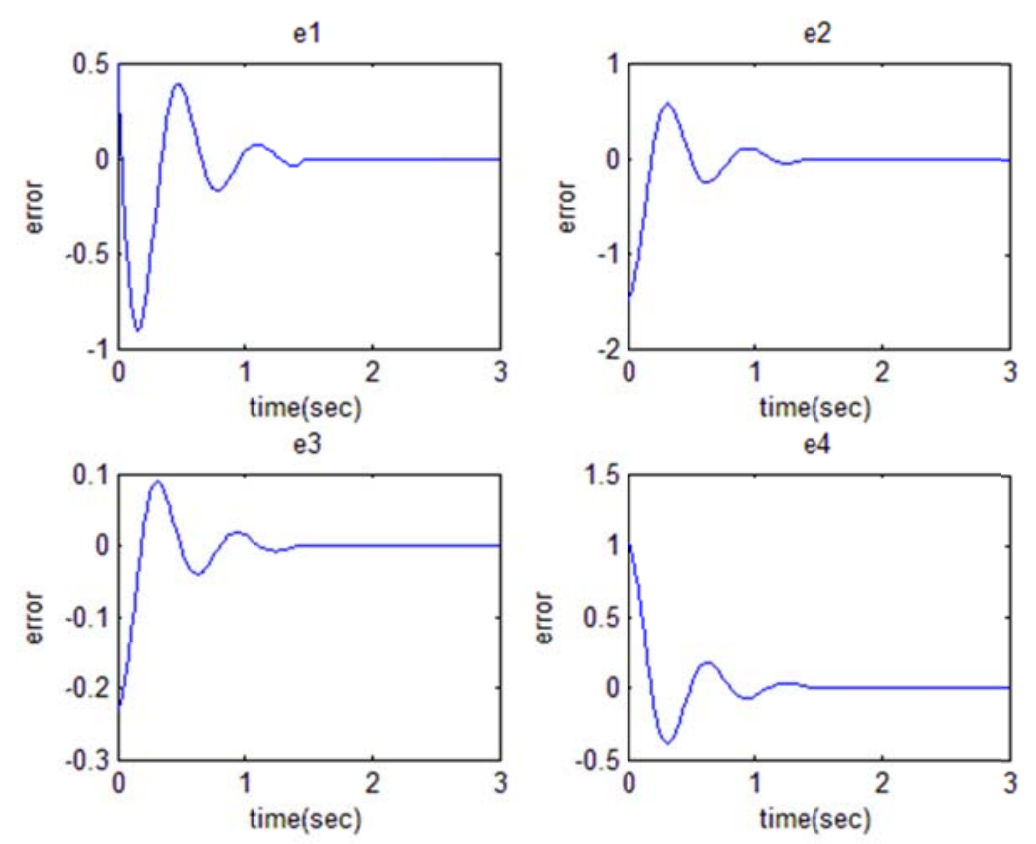

Fig. 1 the observer error dynamics for system (1) 


\section{Conclusions}

This paper deal with the problem of observer design for one-sided Lipschitz nonlinear generalizedsystems based on generalized quadratic stability and Lyapunov function.Sufficient conditions are established in Theorem 1to solve the proposed problem,these conditions are expressed in terms of LMI and LME, which can be easily solved throughefficient numerical software. A simulative example is included to illustrate the effectiveness and advantages of theproposed methods.

\section{Acknowledgemts}

This work was partially supported by the National Natural Science Foundation of China underGrants 60974002 and 61374030.

\section{References}

[1] Barbata A, Zasadzinski M, et al. Exponential Observer for a Class of One-Sided Lipschitz Stochastic Nonlinear Systems. IEEE Transactions on Automatic Control, 2015, 60(1): 259 - 264.

[2] Zhang $\mathrm{W}$, Xie $\mathrm{H}$, et al. Improved results on generalised robust $\mathrm{H}_{\infty}$ filtering for Lipschitz descriptor non-linear systems with uncertainties. Control Theory and Applications, IET. 2015, 9(14): 2107-2114.

[3] Thau F E. Observing the state of non-linear dynamic systems[J]. International Journal of Control, 1973, 17(3): 471-479.

[4] Raghavan S, Hedrick J K. Observer design for a class of nonlinear systems[J]. International Journal of Control, 1994, 59(2): 515-528.

[5] $\mathrm{Hu} G \mathrm{D}$. Observers for one-sided Lipschitz non-linear systems[J]. IMA Journal of Mathematical Control and Information, 2006, 23(4): 395-401.

[6] Abbaszadeh M, Marquez H J. Nonlinear observer design for one-sided Lipschitz systems[C]. American Control Conference (ACC), 2010. IEEE, 2010: 5284-5289.

[7] Zhang W, Su H S, Liang Y, et al. Non-linear observer design for one-sided Lipschitz systems: an linear matrix inequality approach $[\mathrm{J}]$. Control Theory and Applications, IET, 2012, 6(9): 1297-1303.

[8] Lu G, Ho D W C. Generalized quadratic stability for continuous-time singular systems with nonlinear perturbation[J]. IEEE Transactions on Automatic Control, 2006, 51(5): 818-823. 